\section{The extended Delaunay tessellation}

The extended Delaunay tessellation

\author{
Nestor Calvo
}

International Center for Computational Methods in Engineering (CIMEC-INTEC), Universidad Nacional del Litoral and CONICET,

Santa Fe, Argentina

Sergio R. Idelsohn

International Center for Computational Methods in Engineering (CIMEC-INTEC), Universidad Nacional del Litoral and CONICET,

Santa Fe, Argentina

International Center for Numerical Methods in Engineering (CIMNE), Universidad Politécnica de Cataluña, Barcelona, Spain

\section{Eugenio Oñate}

International Center for Numerical Methods in Engineering (CIMNE), Universidad Politécnica de Cataluña, Barcelona, Spain

Keywords Mesh generation, Delaunay/Voronoi tessellations

Abstract The extended Delaunay tessellation (EDT) is presented in this paper as the unique partition of a node set into polyhedral regions defined by nodes lying on the nearby Voronoi spheres. Until recently, all the FEM mesh generators were limited to the generation of tetrahedral or hexahedral elements (or triangular and quadrangular in 2D problems). The reason for this limitation was the lack of any acceptable shape function to be used in other kind of geometrical elements. Nowadays, there are several acceptable shape functions for a very large class of polyhedra. These new shape functions, together with the EDT, gives an optimal combination and a powerful tool to solve a large variety of physical problems by numerical methods. The domain partition into polyhedra presented here does not introduce any new node nor change any node position. This makes this process suitable for Lagrangian problems and meshless methods in which only the connectivity information is used and there is no need for any expensive smoothing process.

\section{Introduction}

Several numerical methods in computational mechanics, as well as other volume integration methods need to subdivide the total domain into sub-domains called "elements". This is the case for the finite element method (FEM) and the finite volume method (FVM).

Once defined the total domain by the boundary surfaces, two standard algorithms may be used to perform the partition:

(1) divide the total domain into elements by an advancing front technique (AFT) (George, 1991; Lohner, 1996), or
583

Received May 2002 Revised December 2002 Accepted January 2003 
EC

$20,5 / 6$

584

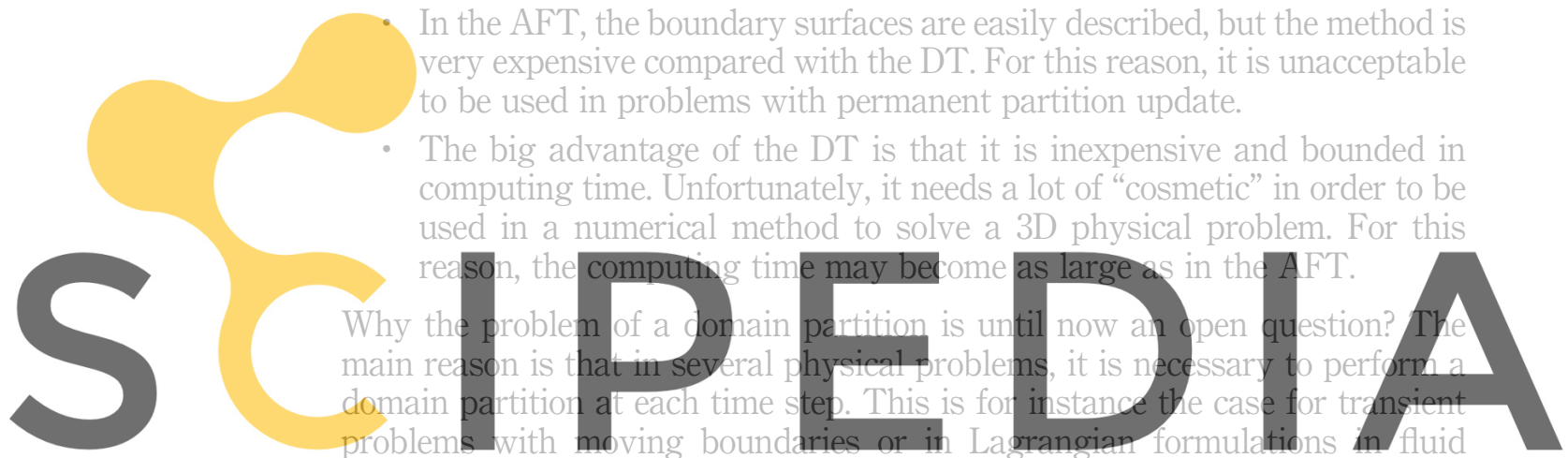

mechanics. In these cases the use of a bounded method is mandatory, the

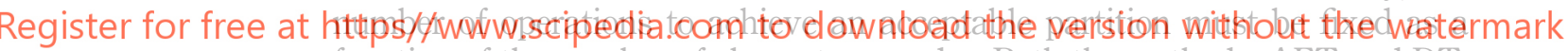
function of the number of elements or nodes. Both the methods, AFT and DT. need a process of cosmetics which increases without a fixed limit in the computing time.

In this paper, a method based on the DT will be described. The problem will be: given a node distribution, find an optimal partition, regarding computing time and element shape, to be successfully used in a numerical method.

It must be noted that obtaining a node distribution in a domain is not a difficult task and it is a bounded operation. The goal of this paper will be to obtain from an arbitrary node distribution a partition of the total domain. This partition will be optimal in order to be used in a numerical method as the FEM and the number of operations to obtain this partition must be bounded with $n^{\beta}$, where $n$ is the total number of nodes and $\beta$ must be much less than 2 . Furthermore, the partition must be unique for a given node distribution.

\section{The DT}

In order to better understand the new procedure, classical definitions will be introduced for three entities: Voronoï diagrams, DT and Voronoï spheres.

Let a set of distinct nodes be: $\mathbf{N}=\left\{n_{1}, n_{2}, n_{3}, \ldots, n_{n}\right\}$ in $\mathbb{R}^{3}$. 
(a) The Voronoi diagram of the set $\mathbf{N}$ is a partition of $\mathbb{R}^{3}$ into regions $V_{i}$ (closed and convex, or unbounded), where each region $V_{i}$ is associated with a node $n_{i}$, such that any point in $V_{i}$ is closer to $n_{i}$ (nearest neighbor) than to any other node $n_{k}$. See Figure 1 for a $2 \mathrm{D}$ representation. There is a single Voronoï diagram for each set $\mathbf{N}$.

(b) A Voronoi sphere within the set $\mathbf{N}$ is any sphere, defined by four or more nodes without any node inside. Such spheres are also known as empty circumspheres.

(c) The DT within the set $\mathbf{N}$ is a partition of the convex hull $\Omega$ of all the nodes into regions $\Omega_{i}$ such that $\Omega=\cup \Omega_{i}$, where each $\Omega_{i}$ is the tetrahedron defined by four nodes of the same Voronoi sphere. DTs of a set $N$ are not unique, but each tessellation is dual to the single Voronoï diagram of the set.

The computing time required for the evaluation of all these three entities is of the order $n^{\beta}$, with $\beta \leq 1.333$. Using a very simple bin organization, the computation time may be reduced to near $n$.

The DT of a set of nodes is non-unique. For the same node distribution,

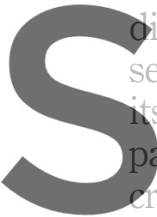

ifferent tetrahedration sensitive to geometric s dual, the Voronoï di partition based on the itical cases of Delaunay inst
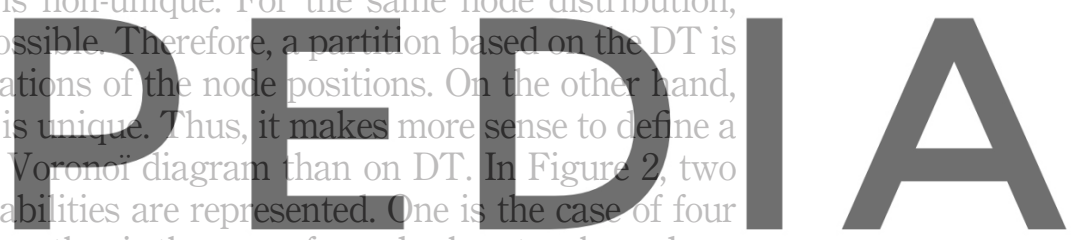

nodes on the same circle and the other is the case of a node close to a boundary.

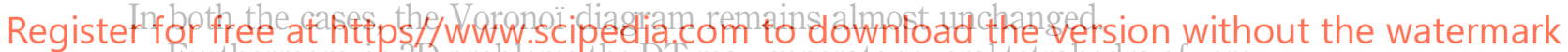
Furthermore, in 31 problems the D1 may generate several tetrahedra of zero or almost zero volume, which introduces large inaccuracies into the shape function derivatives to be used in a numerical method. This is the reason why a DT must be improved iteratively in order to obtain an acceptable partition to be used in a numerical method. The time to obtain an acceptable partition via a DT is then an unbounded operation, thus not satisfying the requirement expressed in the introduction.

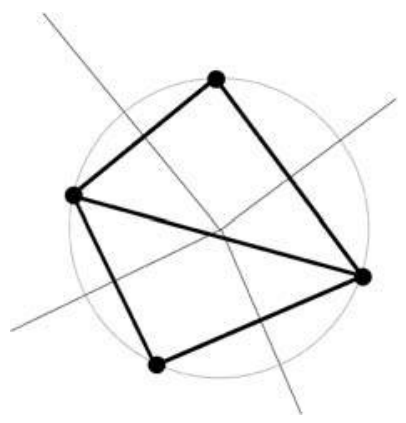

Voronoï Circle

Delaunay Triangulation

Voronoï Diagram

Figure 1.

Voronoï diagram, Voronoï circle and Delaunay triangulation for a four node distribution in $2 \mathrm{D}$ 
EC

$20,5 / 6$

\section{6}

Figure 2.

Instabilities on the DT.

(a) Four nodes on the

\section{same circle; (b) node}

close to a boundary

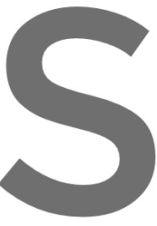

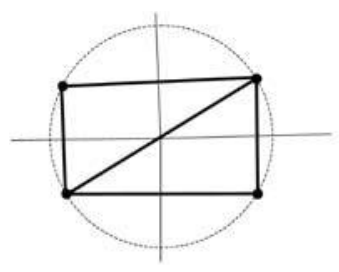

a)
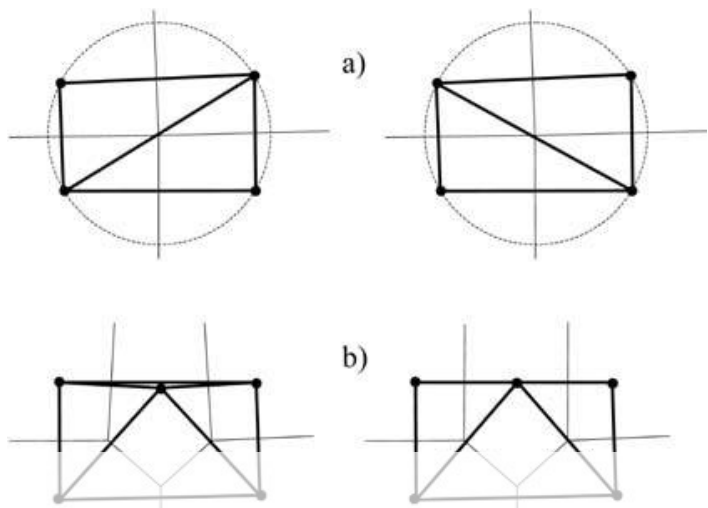

b)

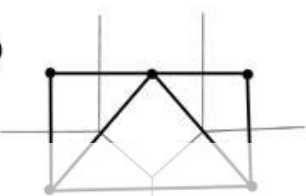

\section{The extended DT}

From the point of view of the application of a domain partition to the solution of a numerical method, the best partition is which the elements have:

(1)

\section{(2)}
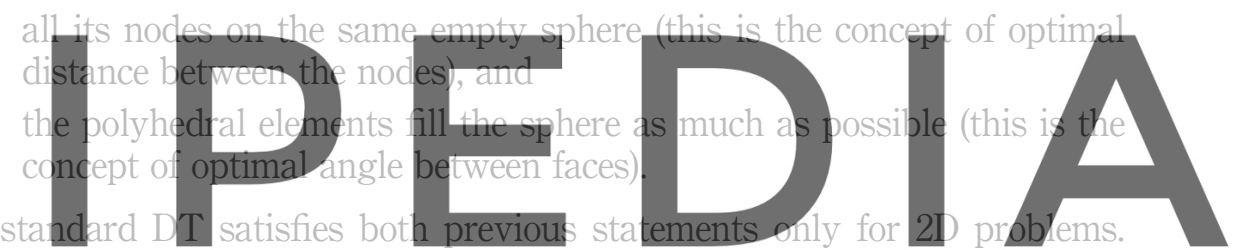

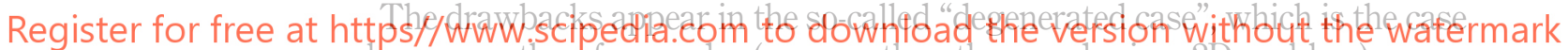

where more than four nodes (or more than three nodes in a 2D problem) are on

the same empty sphere. For instance, in 2D, when four nodes are on the same circumference, two different triangulations satisfy the Delaunay criterion. However, the most dangerous case appears only in 3D. For instance, when five nodes are on the same sphere, five tetrahedra may be defined satisfying the Delaunay criterion, but some of them may have zero or almost zero volumes, called slivers (see Figure 3).

Figure 3.

Five nodes on the same sphere and possible zero or almost zero volume tetrahedron (sliver) on the right

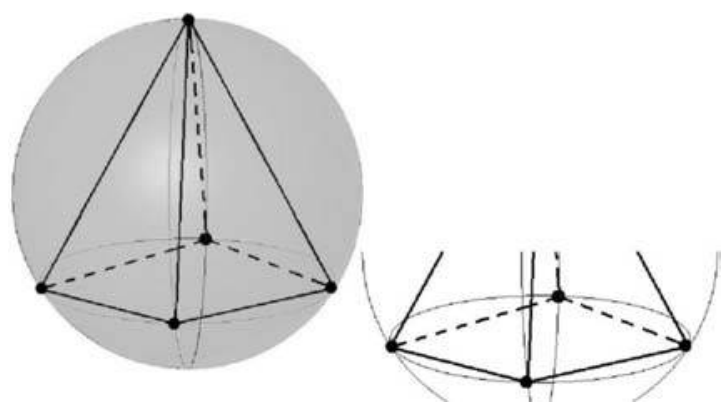


In order to overcome the drawbacks referred, a generalization of the DT will be defined:

Definition. The extended DT (EDT) within the set $\mathbf{N}$ is the unique partition of the convex hull $\Omega$ of all the nodes into regions $\Omega_{i}$ such that $\Omega=\cup \Omega_{i}$, where each $\Omega_{i}$ is the polyhedron defined by all the nodes laying on the same Voronoï sphere.The main difference between the traditional DT and the EDT is that, in the latter, all the nodes belonging to the same Voronoi sphere define a unique polyhedron. With this definition, the domain $\Omega$ will be divided into tetrahedra and other polyhedra, which are unique for a given node distribution, satisfying one of the goals required in the Introduction.

Figure 4, for instance, is a 2D polvgon partition with a triangle, a quadrangle and a pentagon. Figure 5 is a polyhedron with all the nodes on the same sphere, which may appear in a 3D problem.

In order to avoid numerical problems, which may hide polyhedra with more than four nodes, the polyhedra are defined by all the nodes of the same sphere and nearby spheres. The proximity of the spheres is governed by a parameter $\delta$ (see Appendix 1).

The parameter $\delta$ avoids the possibility of having near zero volume

.

retrahedra. When $\delta$ is large, the number of polyhedra with more than four hodes will increase, and the number ot tetrahedra with neatr decrease, and vice versa.
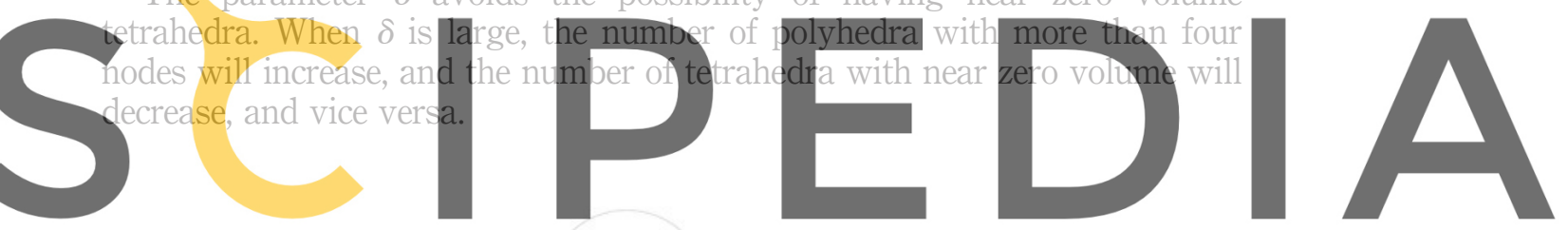

Register for free at https//www.scipedia.com to download the version without the watermark

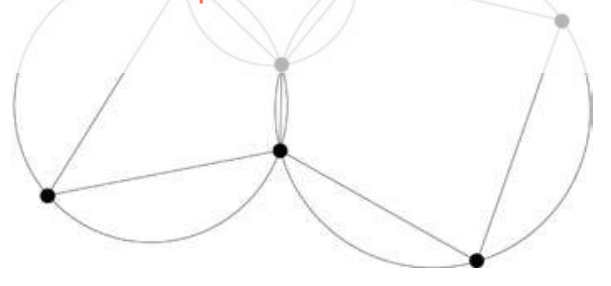

Figure 4.

Two-dimensional partition in polygons.

The triangle, the quadrangle and the pentagon, each are inscribed on a circle
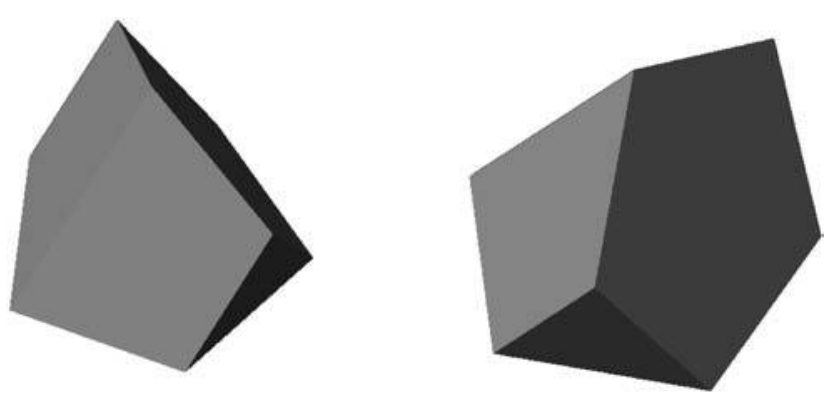

Figure 5.

Eight-node polyhedron. All nodes are on the same sphere 
EC

$20,5 / 6$

\section{8}

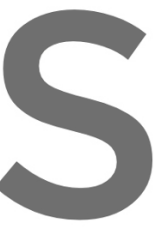

Register for free at
Then, the idea of the EDT is to avoid the tetrahedral partition when more than four nodes are in the same empty sphere (or near the same). When this is the case, the best element to be used in a numerical method is the polyhedron formed with all these nodes.

The EDT allows the existence of a domain partition which:

(1) is unique for a node distribution,

(2) is formed without polyhedra with near zero volume, and

(3) is obtained in a bounded time of order $n$.

Then, it satisfies all the goals stated earlier.

It must be noted that until quite recently, all the mesh generators were limited to the generation of tetrahedral or hexahedral elements (or triangular and quadrangular in 2D problems). The reason for this limitation was the lack of any acceptable shape function to be used in other kind of geometrical elements. Nowadays, several acceptable shape functions for any kind of polyhedron are easily generated (Belikov and Semenov, 1998; Idelsohn et al., 2002 or Appendix 2). These new shape functions, together with the EDT presented in this paper, gives an optimal combination and a powerful tool to solve a large variety of physical problem by

4. The

One of th

domain
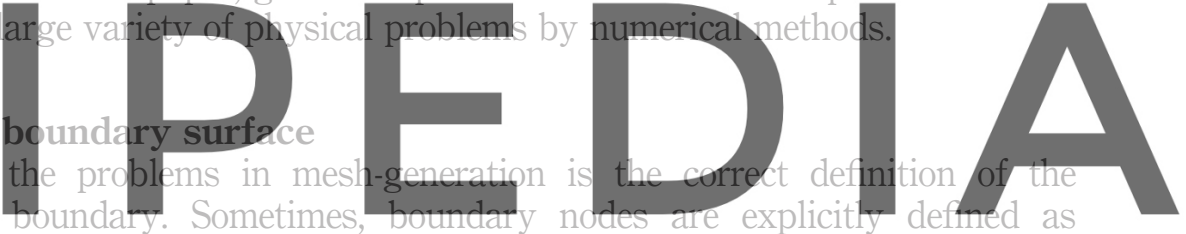

special nodes, which are different from internal nodes. In other cases, the

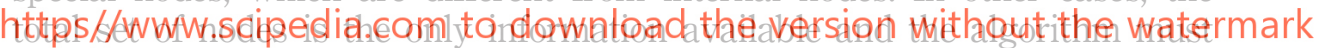

iormitation in fivid mechanics problc node distribution is obtained and the free-surface must be recognized from the node positions.

The use of Voronoï diagrams or Voronoï spheres make it easier to recognize boundary nodes. By considering that the node follows a variable distribution, with $h(\mathbf{x})$ as the minimum distance between two nodes, the following criterion has been used.

All nodes defining an empty circumsphere with a radius $r(\mathbf{x})$ larger than $\alpha$ $h(\mathbf{x})$, are considered as boundary nodes.

In this criterion, $\alpha$ is a parameter close to, but greater than one. Note that this criterion is coincident with the Alpha Shape concept (Akkiraju et al., 1995; Edelsbrunner and Mucke, 1994).

Once a decision has been made concerning which of the nodes are on the boundaries, the boundary surface must be defined. It is well known that, in 3D problems, the surface fitting a number of nodes is not unique. For instance, four boundary nodes on the same sphere may define two different boundary surfaces, one concave and the other convex. 
In order to avoid this undefined boundary, the boundary surfaces will be defined with:

All the polyhedral surfaces having all their nodes on the boundary and belonging to just one polyhedron are boundary surfaces.

The correct boundary surface may be important to define the correct normal external to the surface. Furthermore, in weak forms, the boundary surface is also important for a correct evaluation of the volume domain.

Nevertheless, it must be noted that in the criterion proposed earlier, the error in the boundary surface definition is bounded and proportional to $h$. This is the standard error of the boundary surface definition in a numerical method for a given node distribution.

\section{Element quality indicator}

In order to evaluate the partition obtained with the EDT, compared with other partition as, for instance, DT, a polyhedron quality indicator will be defined.

A wrong mesh is a mesh which is not acceptable to be used in a numerical method. The main drawback of a Delaunay mesh is the presence of zero or almost zero volume polyhedra. In fact, the problem of almost zero volume is the Qxistence of shape function Infinite gradients deteri Classical definition this paper a node di ust be found without close to each other in the node
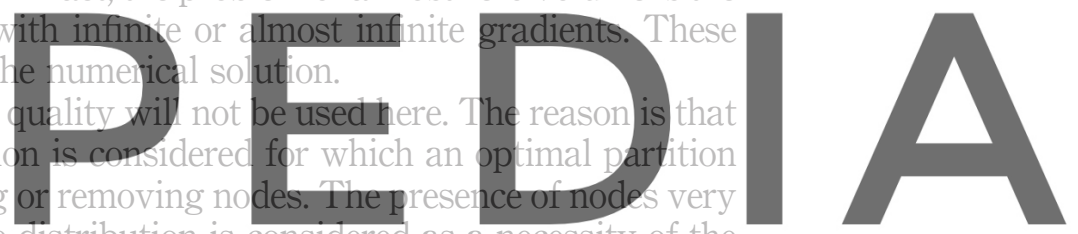

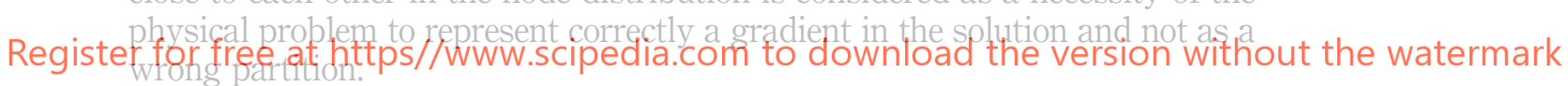

For instance, tetrahedra currently called spires and wedges (see Figure 6) will be considered as elements of good quality because they are the best elements for a node distribution with the nearest nodes along one direction and more separated nodes in the other. These elements represent correctly the gradient "expected" in each direction to solve a particular physical problem. On the other hand, slivers or splinters as well as caps, will be considered as wrong elements because they introduce shape functions with infinite or almost infinite gradients, which are not in agreement with the gradients expected for this node distribution.

In order to obtain a parameter to define which is a good or a wrong element to be used in a numerical method, but considering that the node distribution is fixed and introduced for physical needs, the gradient ratio will be defined as:

$$
\gamma=\frac{\text { maximum gradient of the shape functions }}{\text { maximum gradient expected }}
$$

The maximum gradient expected for the node distribution will be defined in each polyhedron as $1 / h_{\min }$, where $h_{\min }$ is the shortest distance between the two nodes belonging to the polyhedron. 
EC

$20,5 / 6$

590
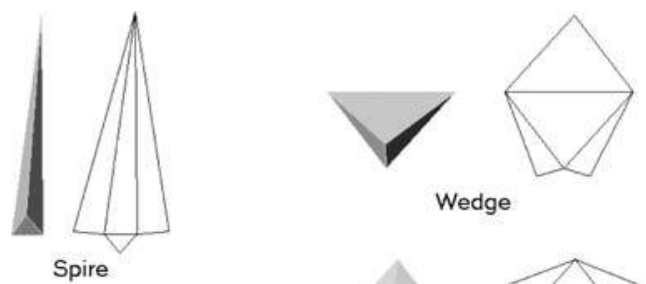

\section{Fionire 6}

Perspective unfolding and naming of some bad-shaped tetrahedra
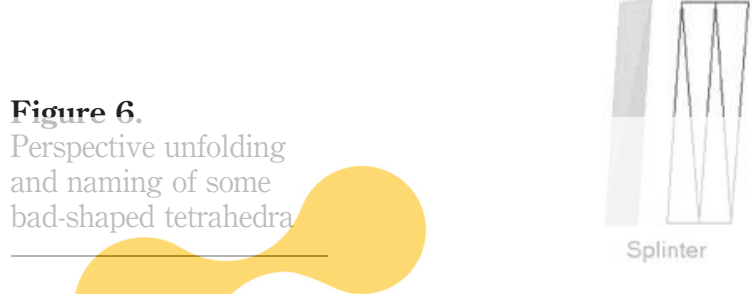

Splinter

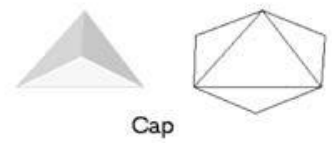

Cap

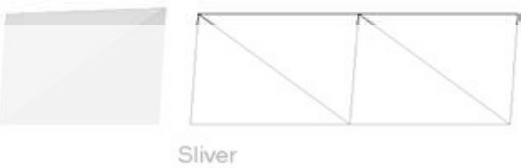

The maximum gradient of the shape functions may be evaluated directly using the largest gradient modulus from the shape functions of the polyhedron $\left|\nabla N_{p}\right|_{\max }$ (see Appendix 2 for the definition of the shape functions $N_{p}$ ).
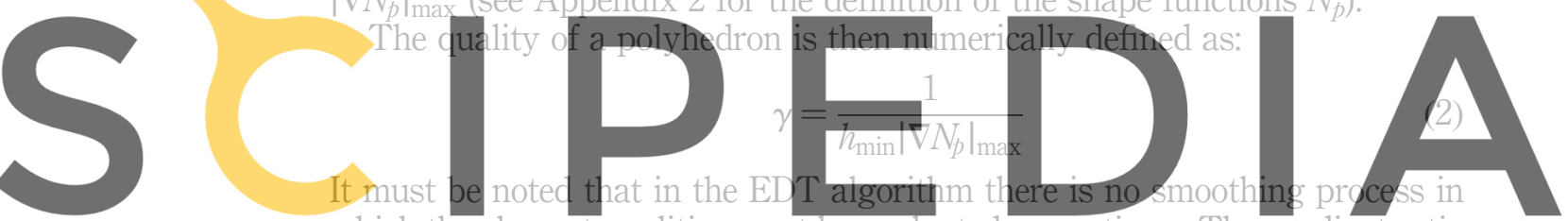

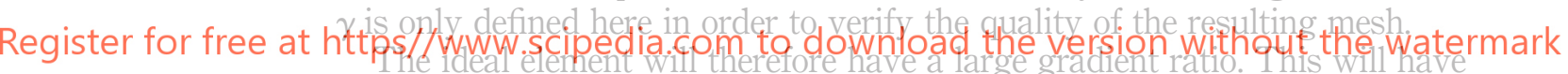

both: the required distribution on the Voronoï sphere and an acceptable shape function for numerical computations. On the other hand, bad elements have all their nodes on the same empty sphere, but the small $\gamma$ value is indicating high shape function gradients capable to destroy the numerical solution.

Taking into account that the computer precision nowadays is of order $10^{-16}$, and also the results from the numerical test performed in the next section, a gradient ratio $\gamma>10^{-2}$ is recommended in order to accept a mesh for numerical computations.

\section{Numerical test}

In order to check if a domain partition is acceptable or not to be used in a numerical method, a physical problem must be solved using a defined partition.

A cube of unit side, with an internal exponential source, has been used to validate the EDT.

The problem to be solved is the classical Poisson equation:

$$
\nabla^{2} u=f(x, y, z)
$$

With the internal source: 


$$
\begin{aligned}
f(x, y, z)= & \left(-2 k y z(1-y)(1-z)+\left(k y z(1-y)(1-z)(1-2 x)^{2}\right)^{2}\right. \\
& -2 k z x(1-z)(1-x)+\left(k z x(1-z)(1-x)(1-2 y)^{2}\right)^{2} \\
& \left.-2 k x y(1-x)(1-y)+\left(k x y(1-x)(1-y)(1-2 z)^{2}\right)^{2}\right) \\
& \times\left(-\mathrm{e}^{k x y z(1-x)(1-y)(1-z)} /\left(1-\mathrm{e}^{k / 64}\right)\right)
\end{aligned}
$$

The extended

Delaunay tessellation

The boundary condition is the unknown function set $u$, equal to zero on all the boundaries.

This problem has the following analytical solution:

$$
u(x, y, z)=\left(1-\mathrm{e}^{k x y z(1-x)(1-y)(1-z)}\right) /\left(1-\mathrm{e}^{k / 64}\right)
$$

Several node distributions have been tested with $125\left(5^{3}\right), 729\left(9^{3}\right), 4,913\left(17^{3}\right)$, and 35,937 $\left(33^{3}\right)$ nodes, with structured and non-structured node distributions. In all cases, the numerical solution was obtained using the linear finite element shape functions for tetrahedral elements and polyhedral shape functions (as defined in the Appendix 2) for polyhedral elements.

.

For the structured no

enerate the nodes. Init

constant distance $h$ bet

een randomly displaced

arbitrary, but structur
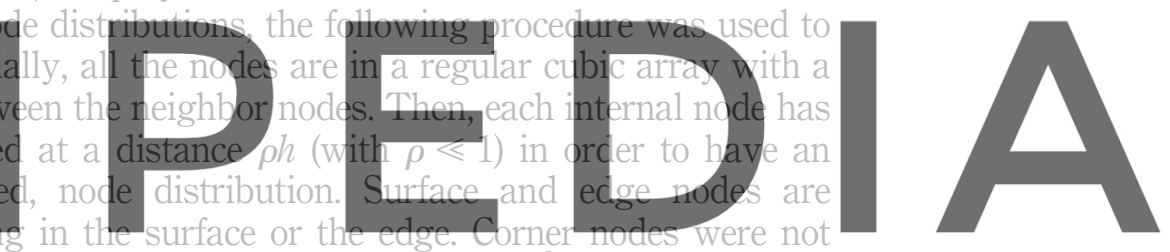

perturbed. In this paper, the parameter $\rho$ was fixed to $10^{-6}$.

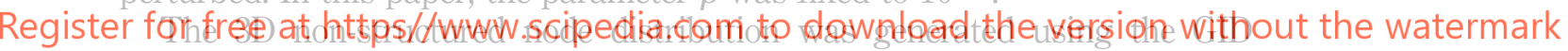

pre/post-processing code (GID, 2002) with a constant $h$ distribution. GID generates the nodes using an $A F T$, which guarantees that the minimal distance between the two nearby nodes lies between $0.707 \mathrm{~h}$ and $1.414 \mathrm{~h}$.

\subsection{EDT versus DT}

It must be noted that in the 2D problems, both node distributions generated as described before, structured and non-structured, will give a Delaunay partition with near-constant area triangles, which is optimal for a numerical solution. Nevertheless, this is not the case in the 3D problems in which, even for a constant $h$ node distribution, many zero or near-zero volume tetrahedra (slivers) will be obtained on a standard Delaunay partition (Edelsbrunner and Damrong, 2001). Figure 7 shows, for instance, the presence of slivers on a structured eight-node distribution. Slivers may introduce large numerical errors in the solution of the unknown functions and their derivatives, which may completely destroy the solution.

In order to show this behavior and to show that the EDT eliminates this problem, the following tests were performed: for a fixed-node partition (e.g. $17^{3}$ nodes) the $\delta$ parameter (introduced in Section 3 and in Appendix 1) was swept 
EC $20,5 / 6$

592

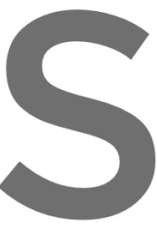

from 0 to $10^{-1}$. With $\delta=0$ (i.e. Voronoï spheres are never joined) the standard DT is obtained. Larger values of $\delta$ give the EDT.

Figure 8 shows the error in $\mathrm{L}_{2}$ norm for the derivative of the solution of the $3 \mathrm{D}$ problem stated in equations (1) and (2). This has been done both for structured and non-structured distributions against the $\delta$ parameter. It can be shown that in both cases the errors are very large $\left(\sim 10^{1}\right)$ for $\delta>10^{-6}$ and very small $\left(\sim 10^{-2}\right)$ for $\delta>10^{-5}$. Larger $\delta$ do not change the results. Hereafter, the EDT will be considered for $\delta>10^{-4}$.

This example is very important because it is showing that, for a given node distribution, a tetrahedration using the standard Delaunay concept do not work. Mesh generators currently use edge-face swapping or another cosmetic algorithms to overcome the presence of wrong elements. All those operations are unbounded in computing time. The idea of joining similar spheres, even for a very small $\delta$ parameter, solves this problem in a very simple way. The wrong tetrahedra are automatically joined to form polyhedra with optimal shapes.

The results of Figure 8 also show that $\delta$ must be large compared with the computer precision (e.g. $\sim 10^{-5}$ for a computer precision of order $10^{-16}$ ) but also means that $\delta$ is not a parameter to be adjusted in each example as results

\section{do not \\ carried \\ All the polyhedra obtain}
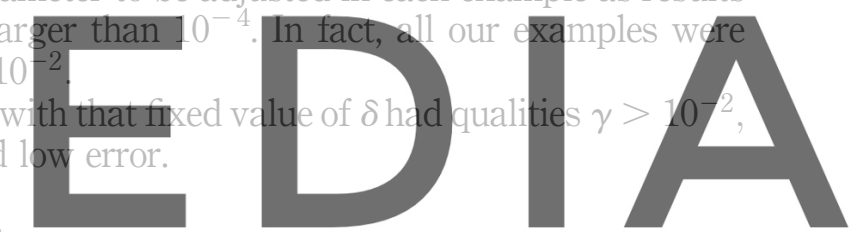

In order to show the performance of the EDT to generate good elements, the

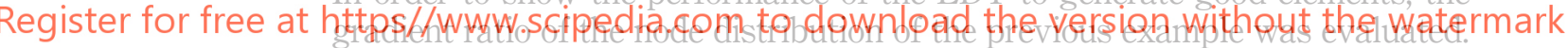

In Figure 9, for a fixed $\delta$, the heights of the columns represent the number of eiements having the same gradient ratio.

The importance of Figure 9 is to show that, by joining similar spheres, the best polyhedra are automatically built. For the standard DT (and also for small $\delta$ values), there are bad tetrahedra for both the structured and the

Figure 7.

Presence of slivers in a Delaunay partition of a perturbed cube. Left: tetrahedra produced by the Delaunay partition. Right: slivers isolated
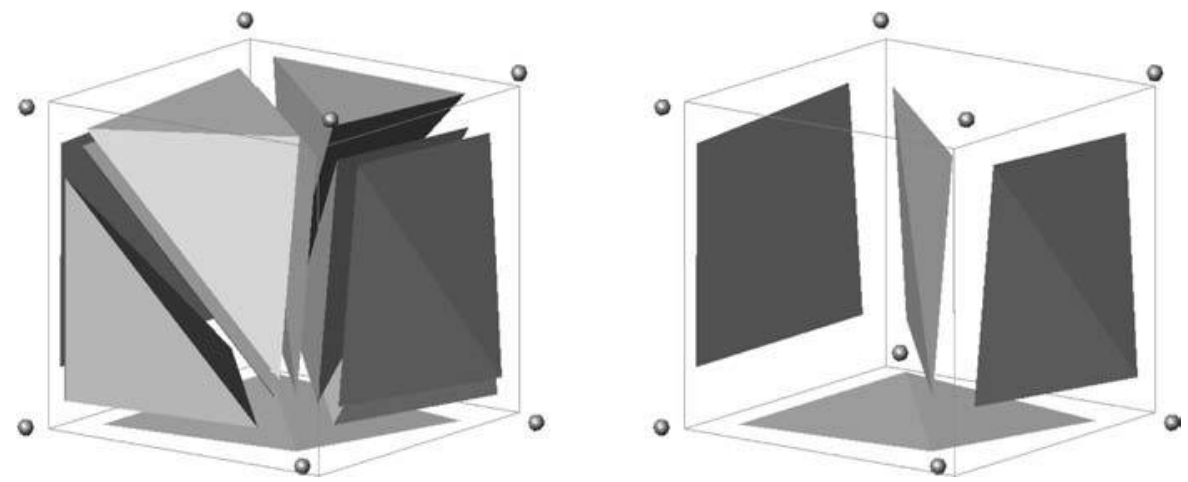


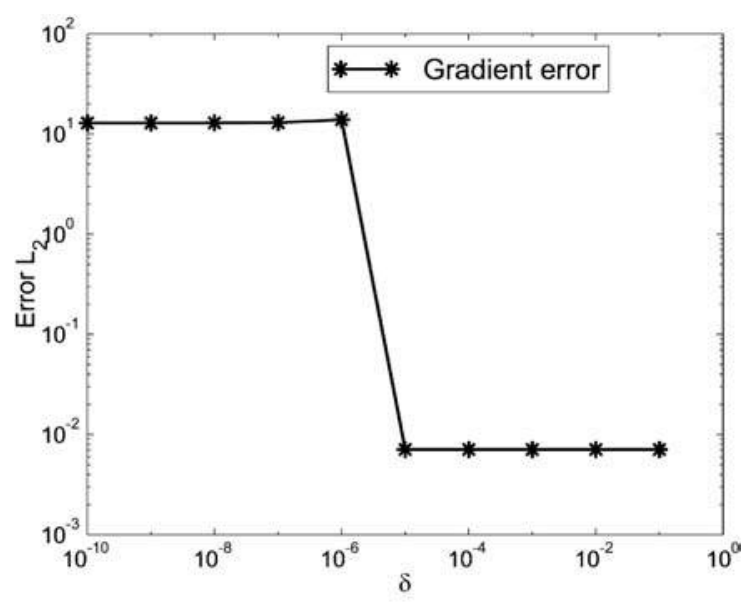

(a)

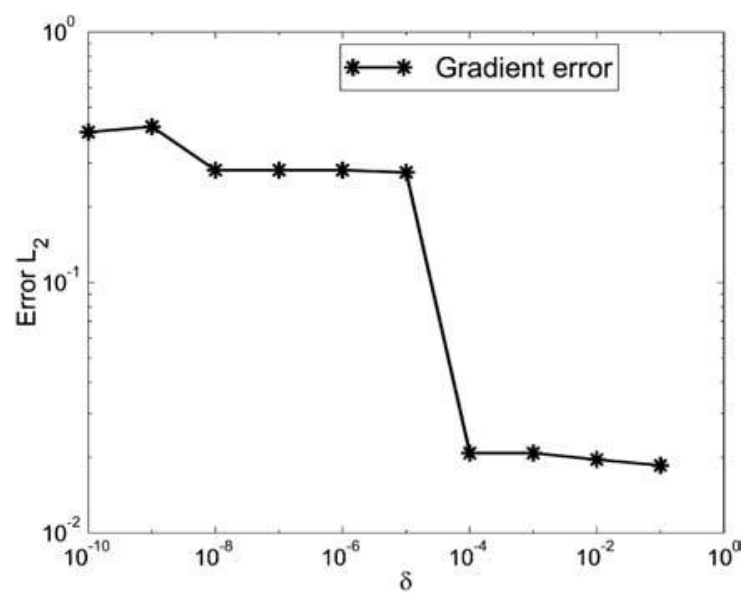

(b)
The extended

Delaunay tessellation

593

Figure 8.

Cube with exponential source. Error of the derivative in $\mathrm{L}^{2}$.

(a) Structured node distribution; (b) non-structured node distribution

non-structured node distribution. Increasing $\delta$, the slivers disappear and, for the structured case, all the elements become "automatically" hexahedra (cubes), which is the optimal tessellation for this node distribution. For the non-structured case, EDT insure a mesh without any sliver when $\delta$ is set to any value larger than $10^{-6}$.

\subsection{Convergence rate}

Figure 10 shows the convergence of the above-defined example, when the number of nodes is increased from $5^{3}$ to $33^{3}$. The upper plots show the error in $L^{2}$-norm, both for the function and its derivatives. All the graphics shows 
EC

$20,5 / 6$

594
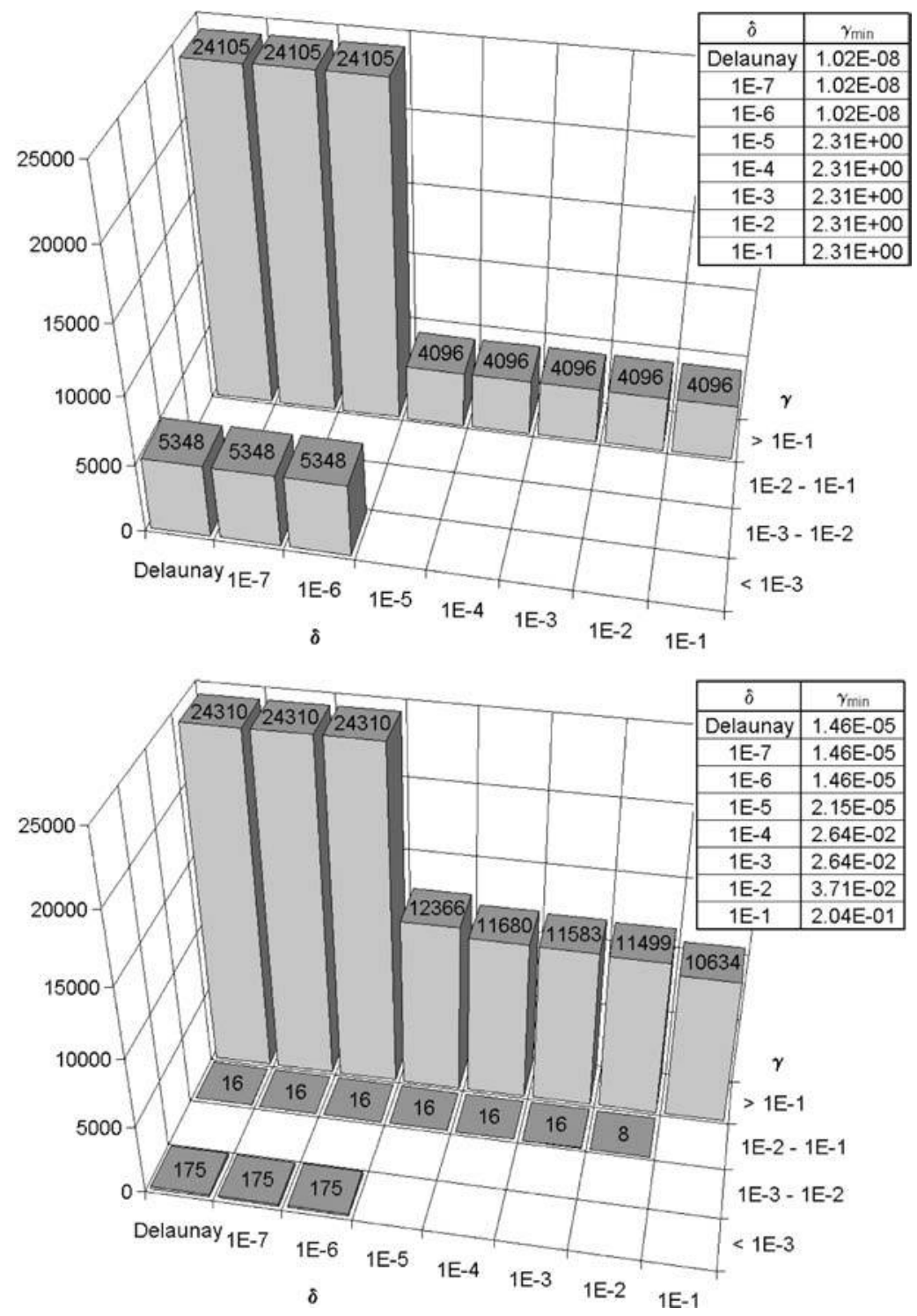

Figure 9.

Number of polyhedra by volume ratio for different $\delta$. Top: structured node distribution. Bottom: non-structured distribution made by GID

an excellent convergence rate. It must be noted that for all the non-structured node distributions tested (and also the structured ones for $\rho=10^{-6}$ ), the FEM with elements generated using a DT gave totally wrong results, and even at times ill-conditioned matrices were frequently found during the stiffness matrix evaluation. 


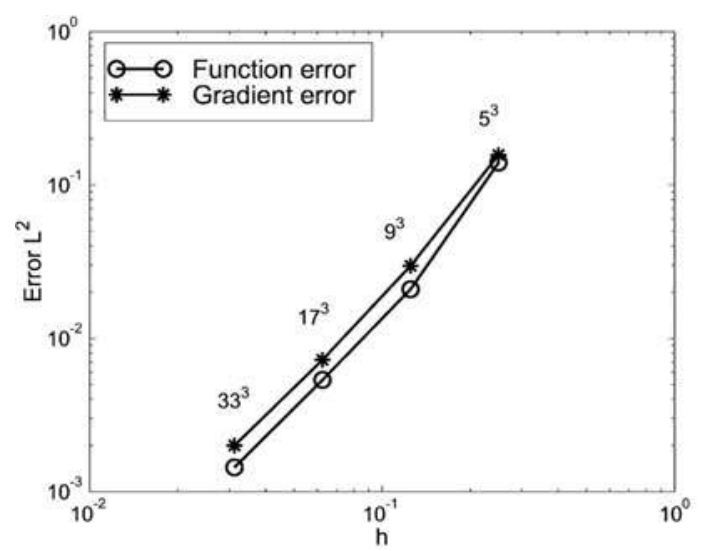

(a)

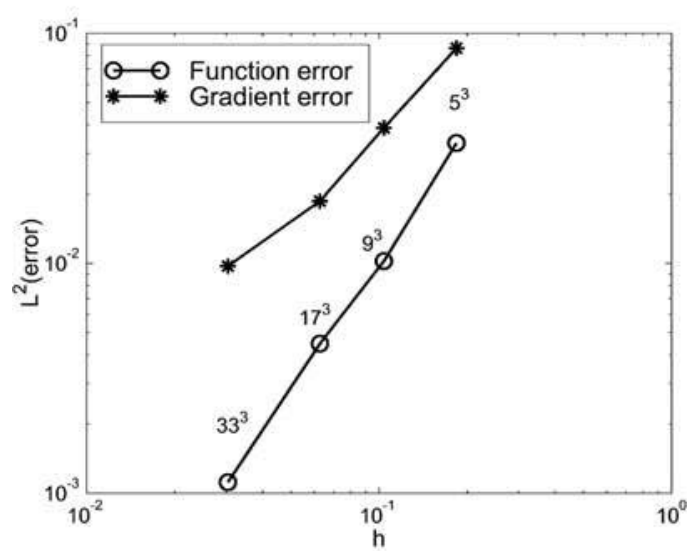

(b)

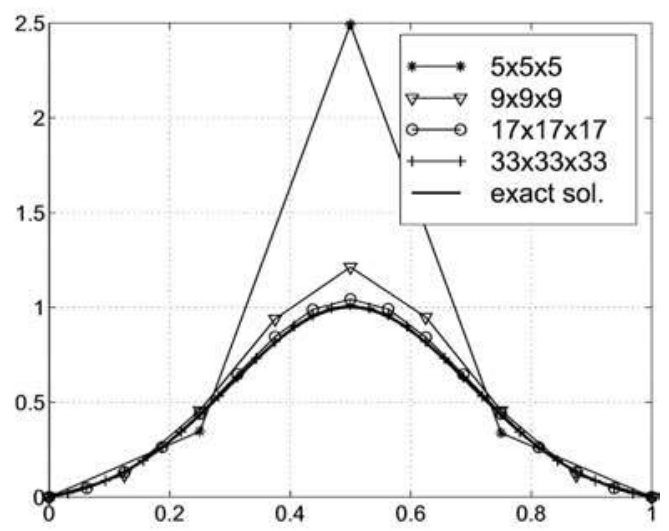

(c)
The extended Delaunay tessellation

595
Figure 10.

Cube with exponential source. Convergence of the numerical solution and its gradient for different partitions. (a) Structured node distribution;

(b) non-structured node distribution;

(c) center-line solutions obtained with structured node distribution (the same results were obtained using the non-structured node distribution) 
EC

$20,5 / 6$

596

\subsection{Computing times}

In order to validate that the number of operations in the evaluation of the EDT partition is of order $n$, the computing time in a standard PC (Intel PIII $800 \mathrm{MHz}$ ) was analyzed. Figure 11 shows the time in seconds for both the structured and the non-structured cases.

A regression of the obtained results shows that the computing time is approximately:

$$
\begin{aligned}
& t(\mathrm{~s})=0.000325 n^{1.08} \quad \text { for structured meshes, and } \\
& t(\mathrm{~s})=0.000283 n^{1.10} \quad \text { for non-structured, }
\end{aligned}
$$

showing that the convergence exponent is even better than the 1.333 bound expected in a DT.

\subsection{Three-dimensional arbitrary geometry mesh}

In order to show the performance of the method with an arbitrary 3D geometry, a volume representing the vocal A was generated with distributions of $n \cong 10^{3}$ and $10^{5}$ nodes, using the GID node generator with a constant $h$. Figure 12 (left) shows, for instance, the boundary node distribution for $n=8,452$ nodes.

Using the EDT algorithm described in Section 3 with the boundary surface definition described in Section 4 and an alpha shape parameter $\alpha=1.3$, polyhedral partitions were found with external boundary surfaces represented

in Figure 12.

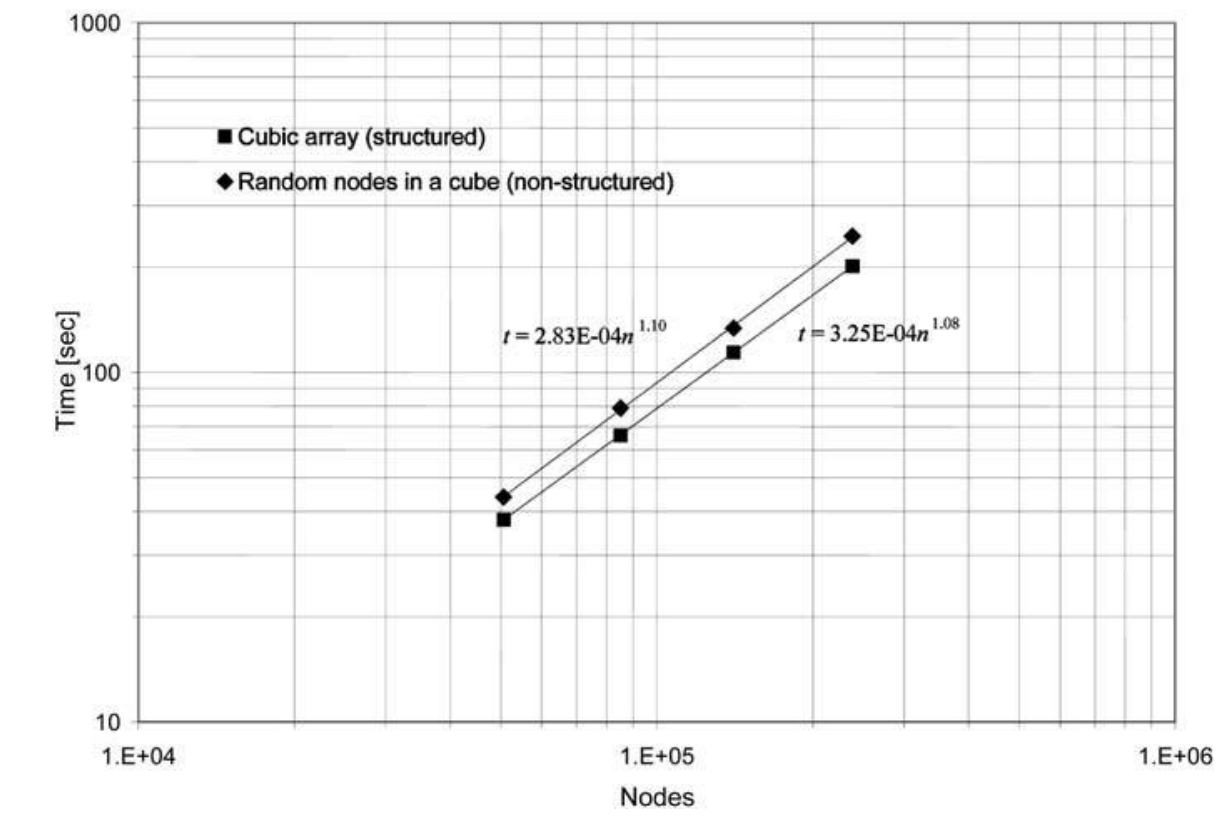

Figure 11.

Time versus number of nodes in a standard PC 
The following are the main characteristics polyhedral mesh made for the larger node set, compared with the tetrahedral mesh obtained via the standard DT (Table I).

From a total of 106,947 nodes, the standard DT generates 599,934 tetrahedra, but 349 of them are slivers. On the other hand, EDT generates 430,262 tetrahedra and 67,162 polyhedra with more than four nodes. None of them have gradient ratio smaller than $10^{-2}$. Another interesting conclusion to take out from this example is that in the EDT mesh, 86.5 percent of the elements are tetrahedral and then, in these elements the definition of the shape functions (Appendix II) will be coincident with classical linear shape functions of the FEMs.

Finally, Figure 13 shows the slivers distribution in the mesh. Elements having a $\gamma$ parameter smaller than $10^{-2}$ have been plotted in black. Zero bad polyhedra have been found in the partition given by the EDT.
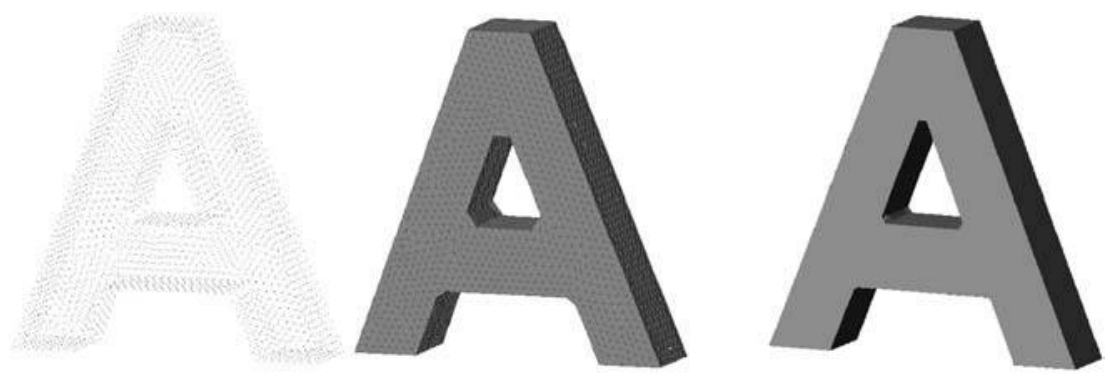

\begin{tabular}{lcc}
\hline & EDT & DT \\
\hline Nodes & 106,947 & 106,947 \\
Polyhedra & 497,424 & 599,934 \\
Tetrahedra & 430,262 & 599,934 \\
Slivers & 0 & 349 \\
$\gamma_{\text {min }}$ & $1.84 \mathrm{e}-2$ & $2.34 \mathrm{e}-16$ \\
\hline
\end{tabular}

The extended Delaunay tessellation

597

Figure 12. Three-dimensional arbitrary geometry. Left: boundary nodes from the point distribution generated by GID with constant $h$ and 8,452 nodes. Center: boundary surface for 8,452 nodes. Right: boundary surface for 106,947 nodes
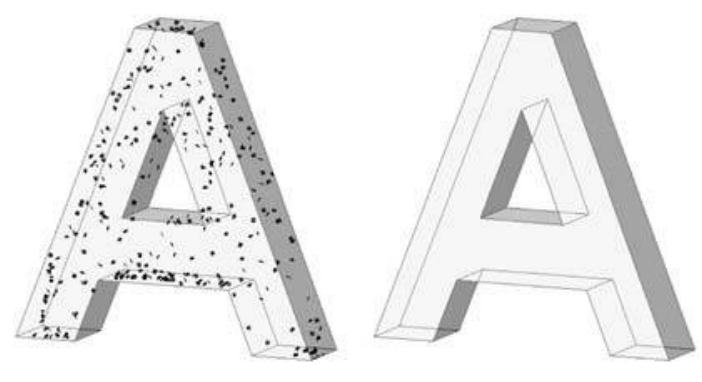

Figure 13.

Wrong elements in the 106,947 nodes tessellation. Left: 349 bad tetrahedral (slivers) in the DT. Right: zero wrong polyhedra in the EDT 
$\mathrm{EC}$ $20,5 / 6$

\section{8}

\section{References}

Akkiraju, N., Edelsbrunner, H., Facello, M., Fu, P., Mücke, E. and Varela, C. (1995), “Alpha shapes: definition and software", Proceedings of the 1st International Computational Geometry Software Workshop, pp. 63-6, url: http://www.geom.umn.edu/software/cglist/ GeomDir/shapes95def/

Belikov, V. and Semenov, A. "Non-Sibsonian interpolation on arbitrary system of points in Euclidean space and adaptive generating isolines algorithm", Numerical Grid Generation in Computational Field Simulation, Proc. of the 6th Intl Conf., July 1998, Greenwich Univ.

Edelsbrunner, H. and Damrong, G. (2001), "An experimental study of sliver exudation", Proceedings 10th International Meshing Roundtable, 7-10 October, Sandia National Laboratories, pp. 307-16.

Edelsbrunner, H. and Mucke, E.P. (1994), “Three-dimensional alpha shapes”, ACM Transactions on Graphics, Vol. 13, pp. 43-72.

George, P.L. (1991), Automatic Mesh Generation, Applications to Finite Methods, ISBN 0-471-93097-0, Wiley, New York.

GID (2002), "The personal pre and post processor”, CIMNE, Barcelona, url: http://gid.cimne.upc.es Idelsohn, S., Oñate, E., Calvo, N. and Del Pin, F. (2002), "The meshless finite element method", International Journal for Numerical Methods in Engineering (in press).

Lohner, R. (1996), "Progress in grid generation via the advancing front technique", Engineering with Computers, Vol. 12, pp. 186-210.

\section{Appendix 1. Criterion to join polyhedra}

Consider two Voronoï spheres having nearby centers. See Figure A1 for a two-dimensional reference.

As both Voronoï spheres are empty, they must satisfy the following relationship: 


$$
\left|r_{2}-r_{1}\right| \leq\left\|c_{1}-c_{2}\right\|
$$

where $r$ are the radii and $c$ the centers of the spheres.

Thus, two spheres are similar when their centers satisfy:

$$
\left\|c_{1}-c_{2}\right\|<\delta r_{\mathrm{rms}},
$$

where $\delta$ is a small non-dimensional value and $r_{\mathrm{rms}}$ is the root mean square radius.

Two polyhedra will be joined if they belong to similar spheres.

The algorithm finds all the four-node empty spheres, and then the polyhedra are successively joined using the above criterion. It must be noted that when all the nodes of a polyhedron belongs to another polyhedron, only the last one is considered.

\section{Appendix 2. Shape functions for arbitrary 3D polyhedra}

For any point within a polyhedron $\mathbf{P}$, there is a Voronoil cell $\mathbf{V}(x)$ associated with the variable point $x$ in the Voronoï tessellation of the set $\mathbf{P} \cup\{x\}$.

Figure A2 shows that every node $n_{p} \in \mathbf{P}$ has a corresponding face $\mathbf{F}_{p}$ of $\mathbf{V}$, which is normal to the segment $\left\{x, n_{p}\right\}$ by its midpoint. This is because $\mathbf{V}$ is the set of points closer to $x$ than any other point.

Defining the functions:
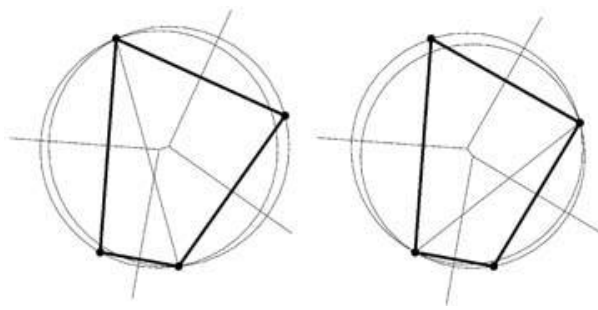

Figure A1.

Four nodes in near-degenerate position showing the empty circumcircles, the Voronoï diagram and the corresponding discontinuous Delaunay triangulation

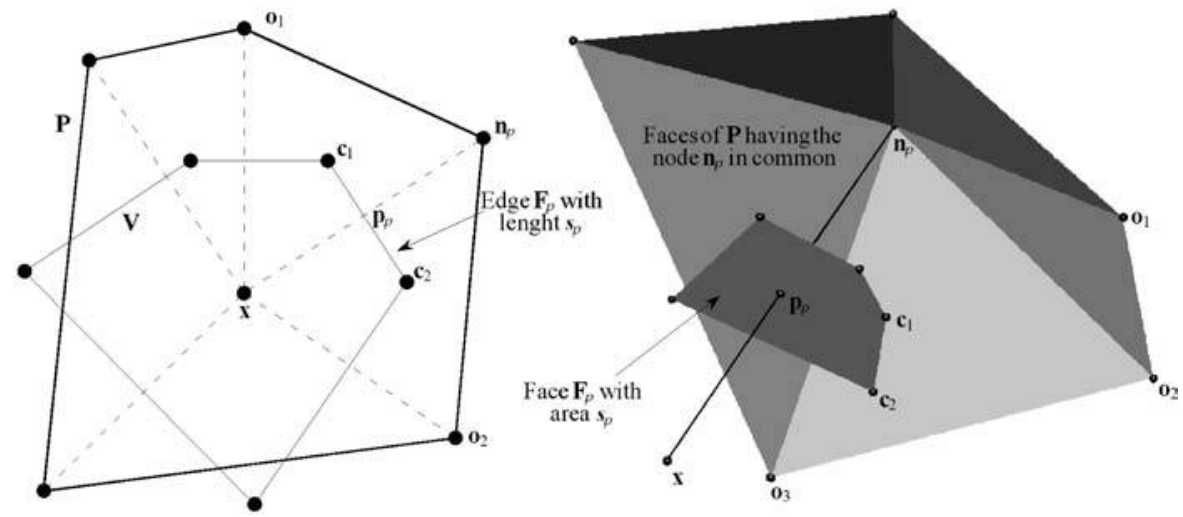

Figure A2.

Elements defining 2D and $3 \mathrm{D}$ shape functions 


$$
\phi_{p}(x)=s_{p} /\left\|n_{p}-x\right\|=s_{p} / h_{p}
$$

as the quotient of the Lebesgue measure $s_{p}$ of $\mathbf{F}_{p}$ and the distance $h_{p}$ between the point $x$ and the node $n_{p}$. The shape functions are:

$$
N_{p}=\phi_{p} / \sum_{q} \phi_{q}
$$

These functions automatically satisfy the partition of unity property:

$$
\sum_{p} N_{p}=1
$$




\section{This article has been cited by:}

1. Amirtham Rajagopal, Markus Kraus, Paul Steinmann. 2018. Hyperelastic analysis based on a polygonal finite element method. Mechanics of Advanced Materials and Structures 25:11, 930-942. [Crossref]

2. Abdiel Ramon Leon Bal, Ulrich Hoppe, Thai Son Dang, Klaus Hackl, Günther Meschke. 2018. Hypoplastic particle finite element model for cutting tool-soil interaction simulations: Numerical analysis and experimental validation. Underground Space 3:1, 61-71. [Crossref]

3. S. de Azevedo, M. F. González, C. Cintas, V. Ramallo, M. Quinto-Sánchez, F. Márquez, T. Hünemeier, C. Paschetta, A. Ruderman, P. Navarro, B. A. Pazos, C. C. Silva de Cerqueira, O. Velan, F. Ramírez-Rozzi, N. Calvo, H. G. Castro, R. R. Paz, R. González-José. 2017. Nasal airflow simulations suggest convergent adaptation in Neanderthals and modern humans. Proceedings of the National Academy of Sciences 114:47, 12442-12447. [Crossref]

4. P.B. Ryzhakov, J. Marti, S.R. Idelsohn, E. Oñate. 2017. Fast fluid-structure interaction simulations using a displacement-based finite element model equipped with an explicit streamline integration prediction. Computer Methods in Applied Mechanics and Engineering 315, 1080-1097. [Crossref]

5. M Mejia-Ugalde, A Dominguez-Gonzalez, M Trejo-Hernandez, LA Morales-Hernandez, RA OsornioRios, JP Benitez-Rangel. 2016. Triangulation intersection approach from Poisson's equation applied to automatic tool selection in computer numerical control mill-lathe. Proceedings of the Institution of Mechanical Engineers, Part B: Journal of Engineering Manufacture 230:4, 722-731. [Crossref]

6. P. Ryzhakov, R. Rossi, A. Viña, E. Oñate. 2013. Modelling and simulation of the sea-landing of aerial vehicles using the Particle Finite Element Method. Ocean Engineering 66, 92-100. [Crossref]

7. Markus Kraus, Amirtham Rajagopal, Paul Steinmann. 2013. Investigations on the polygonal finite element method: Constrained adaptive Delaunay tessellation and conformal interpolants. Computers \& Structures 120, 33-46. [Crossref]

8. Juan Carlos Cante, M. Dolores Riera, Juan Oliver, Jose Manuel Prado, Alvaro Isturiz, C. Gonzalez. 2011. Flow regime analyses during the filling stage in powder metallurgy processes: experimental study and numerical modelling. Granular Matter 13:1, 79-92. [Crossref]

9. Josep Maria Carbonell, Eugenio Oñate, Benjamín Suárez. 2010. Modeling of Ground Excavation with the Particle Finite-Element Method. Journal of Engineering Mechanics 136:4, 455-463. [Crossref]

10. Gerardo V. Mazzetta, Adrián P. Cisilino, R. Ernesto Blanco, Néstor Calvo. 2009. Cranial mechanics and functional interpretation of the horned carnivorous dinosaur Carnotaurus sastrei. Journal of Vertebrate Paleontology 29:3, 822-830. [Crossref]

11. Sergio Ortiz, Damian Siedlecki, Laura Remon, Susana Marcos. 2009. Three-dimensional ray tracing on Delaunay-based reconstructed surfaces. Applied Optics 48:20, 3886. [Crossref]

12. Alexandru Constantiniu, Paul Steinmann, Tom Bobach, Gerald Farin, Georg Umlauf. 2008. The Adaptive Delaunay Tessellation: a neighborhood covering meshing technique. Computational Mechanics 42:5, 655-669. [Crossref]

13. Dimitris Vartziotis, Theodoros Athanasiadis, Iraklis Goudas, Joachim Wipper. 2008. Mesh smoothing using the Geometric Element Transformation Method. Computer Methods in Applied Mechanics and Engineering 197:45-48, 3760-3767. [Crossref]

14. Bo Tang, Junfeng Li, Tianshu Wang. 2008. The least square particle finite element method for simulating large amplitude sloshing flows. Acta Mechanica Sinica 24:3, 317-323. [Crossref] 
15. E. Oñate, J. Rojek, M. Chiumenti, S.R. Idelsohn, F. Del Pin, R. Aubry. 2006. Advances in stabilized finite element and particle methods for bulk forming processes. Computer Methods in Applied Mechanics and Engineering 195:48-49, 6750-6777. [Crossref]

16. E. Oñate, J. García, S.R. Idelsohn, F. Del Pin. 2006. Finite calculus formulations for finite element analysis of incompressible flows. Eulerian, ALE and Lagrangian approaches. Computer Methods in Applied Mechanics and Engineering 195:23-24, 3001-3037. [Crossref] 